\title{
Searches for WIMP annihilation with GLAST
}

\author{
Larry Wai \\ Stanford Linear Accelerator Center, Stanford, California \\ representing the GLAST LAT Collaboration
}

\begin{abstract}
We describe signatures for WIMP annihilation in the gamma ray sky which can be observed by the GLAST mission, scheduled for launch in 2007. We review the search regions, which range from galactic substructure in the Milky Way all the way out to cosmological sources.
\end{abstract}

Key words: GLAST; WIMP; annihilation

\section{Introduction}

Detection of WIMPs by direct or indirect means is challenging, to say the least. Direct detection depends upon the local density of WIMP particles, whereas indirect detection depends upon the density (squared) of WIMP particles in regions of the galaxy or beyond. Indirect detection also depends upon WIMPs being either Majorana particles or a mixture of roughly equal numbers of WIMPs and anti-WIMPs. It is not uncommon for particle physics motivated candidates for WIMPs, such as the SUSY neutralino $(1 ; 2)$, to be Majorana particles, so this is not an unreasonable assumption.

Direct detection, based upon WIMP-nucleon elastic scattering, is the focus of much of the WIMP search effort, and is covered at great length by other experimentalists at this conference. Indirect detection experiments are a motley crew, with signatures highly dependent upon the dark matter distribution and particle type/detector. Indirect experiments can be categorized by particle type which include neutrinos, anti-matter, and gamma-rays (3). Here we focus on the GLAST detector and how other experiments may relate to GLAST in terms of the WIMP annihilation search program.

The GLAST Large Area Telescope (LAT) (4) consists of a tracking detector, calorimeter, and anti-coincidence detector. The tracking detector is composed 
of 36 layers of tungsten and silicon strip detectors, and serves as the target for converting the gamma rays as well as measuring the tracks of the conversion electron-positron pair. The calorimeter is composed of 8 layers of stacked Cesium Iodide crystal logs readout with PIN photodiodes at both ends of each log. The anti-coincidence detector is composed of overlapping plastic scintillator tiles readout with PMTs via wavelength shifting fibers. At the time of writing of this paper, final flight hardware modules are in production, and preparation for integration and testing of the LAT is in full swing. The launch of GLAST into low earth orbit is on schedule for early 2007.

The LAT is designed to present an effective area of $\sim 10000$ square centimeters with a field of view covering $\sim 1 / 6$ of the full sky. The standard mode of operation will be a continuous scan of the sky. The energy threshold is 20 $\mathrm{MeV}$ and gamma ray energies up to $300 \mathrm{GeV}$ can be measured. The angular resolution for the LAT is significantly better than that of EGRET (factor of at least a few), as is the energy resolution, especially above $10 \mathrm{GeV}$. The mission lifetime is designed for 5 years.

The resulting point source sensitivity $\left(\sim 3 \times 10^{-9} \mathrm{~cm}^{-2} \mathrm{~s}^{-1}\right)$ is $\sim 30$ times better than that of EGRET.

WIMPs are expected to form high density clumps according to N-body simulations of test particles with only gravitational interactions $(5 ; 6)$. These high density clumps will enhance WIMP annihilations as the square of the density, and for a wide range of clump distances/sizes (7) the WIMP annihilation flux will appear as a gamma ray point source. Of course for very close and/or large clumps the WIMP annihilation flux can appear as an extended gamma ray source.

\section{GLAST WIMP Signatures}

The main gamma-ray signatures for WIMP annihilation are:

- lack of variability

- $\pi^{0}$ decay energy spectrum (i.e. peak at $67.5 \mathrm{MeV}$ )

- cutoff of energy spectrum, line (i.e. at mass of WIMP)

- inverse Compton scattering of electrons from $\pi^{+/-}$decay

Variability depends upon careful experimental study of instrumental effects so that one can say with certainty whether a particular point source shows any evidence of variability or not. The main background removed from the WIMP annihilation search with this signature are AGNs. 
Search for evidence of the $\pi^{0}$ decay energy spectrum depends upon a careful experimental measurement of the energy spectrum below $\sim 100 \mathrm{MeV}$. This is formidable for two reasons: 1) poor reconstruction of the electron/positron pair tracks at low energies, and 2) systematic errors in the point spread function at low energies combined with systematic errors in the galactic diffuse model. If the experimental obstacles can be overcome, then the main background removed from the WIMP annihilation search will be pulsars.

Search for a cutoff in the energy spectrum, or better still, observation of the suppressed gamma ray "line" at the mass of the WIMP, requires good energy resolution at high energy (10's to 100's of GeV). Systematic errors due to leakage and dead material are the experimental obstacles here and can be studied with a dedicated calibration beam test of LAT modules on the ground. Cutoff of the energy spectrum at the mass of the WIMP will contribute to deviation from power law spectral fits, which are typical of astrophysical sources.

Inverse Compton scattering of electrons from $\pi^{+/-}$decay produces a large ( $\sim 10 \mathrm{deg}$ ) extended source of gamma rays (8). The experimental challenges here are measuring the diffuse emission $(9 ; 10)$ and understanding what is expected from galactic diffuse emission mechanisms. Sophisticated models are available, e.g. the semi-analytic treatment of (11) and the fully numerical GALPROP model $(12 ; 13)$, but clearly this is an area in which GLAST should make a large impact.

\section{GLAST WIMP Search Regions}

The GLAST WIMP search can be conveniently categorized by source location:

- galactic center

- galactic satellites

- galactic halo

- extra-galactic

The EGRET point source located at the galactic center (14) is a potential source of WIMP annihilation. If Sgr A* is a counterpart of the the EGRET point source, then the radio emission has a WIMP annihilation interpretation as synchrotron emission from the electrons from $\pi^{+/-}$decay (15). Some controversy as to actual location of the EGRET point source exists (16). GLAST should make a significant impact in this area by improving the point source location error from 10 arcmin to 30 arcsec. Atmospheric cherenkov telescopes (ACTs) have searched for gamma ray emission from the galactic center, and some tantalizing hints have been observed (17). Clearly, improvement in ACT observations will have a large impact on interpretation of the EGRET point 
source.

Dwarf galaxies are galactic satellites with a known large amount of dark matter. However, cosmological N-body simulations predict significantly more clumps of dark matter than can be associated with the known galactic satellites. Thus there could exist a significant number of "invisible" galactic satellites in the form of large mass $\left(>10^{7}\right.$ solar mass) pure dark matter clumps (18). The possibility for observing WIMP annihilation from dwarf galaxies such as Draco has been studied (19), as well as from the "invisible" galactic satellites predicted by N-body simulation $(18 ; 8)$. A careful study of the WIMP annihilation signatures outlined above with GLAST should be able to determine whether some of the unidentified EGRET point sources (20), or new point sources discovered by GLAST, are the annihilating cores of "invisible" galactic satellites (8).

WIMP annihilation in the "smooth" part of the galactic halo (i.e. the regions between the galactic center and galactic satellites) can also be observed (21). Of course, the difficulty here is understanding what one expects in the galactic diffuse emission due to more "standard" non-WIMP processes. In general, clumpiness in galactic halos has the effect of enhancing WIMP annihilation flux (22).

One exciting possibility is that WIMP annihilation from all extragalactic dark matter contributes some significant fraction to the extragalactic diffuse emission (23). Due to the large flux of extragalactic gamma rays observed by EGRET (10), GLAST may be able to discern spectral features which would be a "smoking gun" for WIMP annihilations.

\section{Summary}

In summary, searches for WIMP annihilation in the gamma ray sky are complementary to the direct detection experiments. Indirect detection of WIMPs (or stringent limits as the case may be) in the gamma ray sky offers unique information on the nature of WIMPs, i.e. Dirac or Majorana, WIMP mass. Furthermore, WIMP annihilation gamma rays could potentially provide unique direct "imaging" of dark matter substructure in the Milky Way.

The GLAST WIMP search program has a number of potential search regions ranging from galactic substructure in the Milky Way all the way out to cosmological sources. If WIMP annihilation gamma rays are indeed collected by GLAST, then timing, spectral, and spatial signatures unique to WIMP annihilation are available which can facilitate rejection of other possible astrophysical sources of gamma rays. 
If GLAST observes a point source with signatures of WIMP annihilation, then ground based gamma ray telescopes can view the point source and search for spectral signatures at or just below the mass of the WIMP. The large effective area of the ground based telescopes at the highest energies make them ideal for identifying the suppressed "line" at the mass of the WIMP.

\section{References}

[1] For reviews see G. Jungman, M. Kamionkowski and K. Griest, Phys. Rep. 267, 195 (1996); L. Bergström, Rept. Prog. Phys. 63, 793 (2000).

[2] P. Gondolo, J. Edsjö, L. Bergström, P. Ullio, and E. A. Baltz, astro-ph/0012234; P. Gondolo, J. Edsjö, P. Ullio, L. Bergström, M. Schelke and E. A. Baltz, astro-ph/0211238; http://www.physto.se/ edsjo/darksusy/.

[3] J. E. Gunn, B. W. Lee, I. Lerche, D. N. Schramm and G. Steigman, Astrophys. J. 223, 1015 (1978); F. W. Stecker, Astrophys. J. 223, 1032 (1978); J. Silk and M. Srednicki, Phys. Rev. Lett. 53, 624 (1984); J. Silk and H. Bloemen, Astrophys. J. 313, L47 (1987); G. Lake, Nature 346, 39 (1990); L. Bergström, P. Ullio and J. H. Buckley, Astropart. Phys. 9, 137 (1998).

[4] W.B. Atwood et. al., NIM A342, 302 (1994); E.D. Bloom et. al., Space Sci.Rev. 75, 109 (1996); N. Gehrels and P. Michelson, Astropart. Phys. 11, 277 (1999)

[5] J. F. Navarro, C. S. Frenk and S. D. M. White, Astrophys. J. 490, 493 (1997).

[6] B. Moore, T. Quinn, F. Governato, J. Stadel and G. Lake, Mon. Not. R. Astron. Soc. 310, 1147 (1999).

[7] S. Ghigna, B. Moore, F. Governato, G. Lake, T. Quinn and J. Stadel, Mon. Not. R. Astron. Soc. 300, 146 (1998).

[8] E. A. Baltz and L. Wai, astro-ph/0403528

[9] S. D. Hunter et al., Astrophys. J. 481, 205 (1997).

[10] P. Sreekumar et al., Astrophys. J. 494, 523 (1998).

[11] D. Maurin, F. Donato, R. Taillet and P. Salati, Astrophys. J. 555, 585 (2001).

[12] A. W. Strong and I. V. Moskalenko, Astrophys. J. 509, 212 (1998).

[13] A. W. Strong, I. V. Moskalenko and O. Reimer, Astrophys. J. 537, 763 (2000).

[14] H. A. Mayer-Hasselwander et al., Astron. \& Astrophys. 335, 161 (1998).

[15] V. Berezinsky, A. V. Gurevich and K. P. Zybin, Phys. Lett. B 294, 221 (1992); V. Berezinsky, A. Bottino and G. Mignola, Phys. Lett. B 325, 136 (1994); P. Gondolo, Phys. Lett. B 494, 181 (2000); G. Bertone, G. Sigl and J. Silk, Mon. Not. R. Astron. Soc. 326, 799 (2001); R. Aloisio, P. Blasi and A. V. Olinto, astro-ph/0402588 
[16] D. Hooper and B. Dingus, astro-ph/0210617

[17] K. Kosack for the VERITAS Collaboration, astro-ph/0403422

[18] P. Blasi, A. V. Olinto and C. Tyler, Astropart. Phys. 18, 649 (2003).

[19] C. Tyler, Phys. Rev. D 66, 023509 (2002).

[20] P. L. Nolan et al., Astrophys. J. 459, 100 (1996); B. L. Dingus et al., Astrophys. J. 467, 589 (1996); P. Sreekumar et al., Astrophys. J. 464, 628 (1996); Y. C. Lin et al., Astrophys. J. Suppl. Ser. 105, 331 (1996); R. C. Hartman et al., Astrophys. J. Suppl. Ser. 123, 79 (1999).

[21] F. Stoehr et. al., Mon. Not. R. Astron. Soc. 345, 1313 (2003).

[22] L. Bergström, J. Edsjö, P. Gondolo and P. Ullio Phys. Rev. D 59, 043506 (1999); E. A. Baltz, C. Briot, P. Salati, R. Taillet and J. Silk, Phys. Rev. D 61, 023514 (2000); C. Calcáneo-Rodán and B. Moore, Phys. Rev. D 62, 123005 (2000); A. Tasitsiomi and A. V. Olinto, Phys. Rev. D 66, 083006 (2002); R. Aloisio, P. Blasi and A. V. Olinto, Astrophys. J. 601, 47 (2004); S. M. Koushiappas, A. R. Zentner and T. P. Walker, Phys. Rev. D 69, 043501 (2004).

[23] P. Ullio, L. Bergström, J. Edsjö and C. Lacey, Phys. Rev. D 66, 123502 (2002). 